Revisión

\title{
Control y prevención de nematodosis en pequeños rumiantes: antecedentes, retos y perspectivas en México
}

David Emanuel Reyes-Guerrero ${ }^{a}$

Agustín Olmedo-Juárez ${ }^{\text {a }}$

Pedro Mendoza-de Gives ${ }^{a^{*}}$

${ }^{a}$ Instituto Nacional de Investigaciones Forestales Agrícolas y Pecuarias. Centro Nacional de Investigación Disciplinaria en Salud Animal e Inocuidad. Unidad de Investigación en Helmintología. Boulevard Paseo Cuauhnahuac No. 8534, Col. Progreso, 62550, Jiutepec, Morelos, México.

*Autor de correspondencia:pedromdgives@yahoo.com; mendoza.pedro@inifap.gob.mx

\section{Resumen:}

En esta revisión se presenta un panorama general de las nematodosis en pequeños rumiantes en México; así como los principales problemas que enfrentan los productores para mantener su actividad productiva. Del mismo modo, se muestra información general sobre las nematodosis gastrointestinales y sus efectos en la salud animal y en la productividad. Por otra parte, se analizan los principales retos o desafíos que se enfrenta el sector pecuario para contrarrestar estas importantes enfermedades, haciendo énfasis en las distintas estrategias de control y prevención incluyendo la quimioterapia, resistencia antihelmíntica, manejo del pastoreo, desparasitación selectiva, estrategia nutricional proteica, vacunación, selección de animales genéticamente resistentes a los nematodos, el uso de plantas y compuestos con actividad nematicida; así como los resultados más recientes en cuanto al uso de hongos nematófagos como agentes de control biológico, entre otras herramientas de control. En este trabajo se hace mención a importantes resultados obtenidos en la investigación generada en el Área de Helmintología del CENID-SAI del INIFAP y se plantea como perspectiva el establecimiento de un método integral de control de estas enfermedades a las distintas fases de desarrollo de los parásitos como "blancos" de ataque hacia dónde dirigir las estrategias de control, a los diferentes estadios evolutivos de estos parásitos, con lo que se esperan los mejores resultados contra 
este grupo de parásitos que tanto afectan a la salud de los rebaños y a la economía de los productores.

Palabras clave: Nematodosis, Parásitos, Ovinos, Caprinos, Control, Prevención.

Recibido: 06/11/2020

Aceptado: 19/01/2021

\section{Introducción}

\section{Importancia de la producción ovina y caprina nacional}

La producción de pequeños rumiantes en México, proporciona un importante aporte de proteína de origen animal para los mexicanos ${ }^{(1)}$, genera 50 mil empleos directos e indirectos para aproximadamente 400 mil familias $^{(2)}$. No obstante, la mala calidad de los pastos $^{(3)}$, los elevados costos de los alimentos ${ }^{(4)}$, las sequías e inundaciones y otros desastres derivados del cambio climático ${ }^{(5)}$, junto con un grupo de nematodiasis amenazan la salud animal y la economía de los productores.

\section{Nematodosis gastrointestinales en pequeños rumiantes}

Los nematodos gastrointestinales (NGI) son gusanos cilíndricos que habitan el tracto digestivo de rumiantes y son considerados como parásitos de gran importancia en la industria ganadera principalmente en sistemas extensivos, tanto de climas tropicales como templados $^{(6)}$. Los parásitos adultos copulan y se produce una gran cantidad de huevos que salen al medio junto con las heces en donde desarrollan hasta larvas infectantes (L3) que contaminan los pastos. Los animales se infectan al consumir el pasto contaminado con estas larvas ${ }^{(7)}$. Los principales NGI en pequeños rumiantes en México son: Haemonchus contortus, Trichostrongylus colubriformis, T. axei, Teladorsagia (Ostertagia) circumcincta, Cooperia spp., Oesophagostomum, Trichuris ovis, Strongyloides papillosus and Bunostomum spp. ${ }^{(8,9)}$. Estos parásitos se presentan generalmente de manera simultánea provocando un cuadro clínico con mayor o menor grado de severidad, dependiendo en gran medida de la edad y del estado nutricional de los animales ${ }^{(10)}$. Haemomchus contortus es considerado uno de los nematodos de mayor patogenicidad en rebaños ovinos y caprinos debido a sus hábitos de hematofagia y a su alta prolificidad. La enfermedad causada por este nematodo es conocida como "hemoncosis" y provoca pérdidas de peso, falta de apetito, disminución de la condición corporal, anemia, debilidad, emaciación, edemas de regiones bajas del cuerpo, susceptibilidad a otras enfermedades y la muerte en animales jóvenes ${ }^{(11)}$. 
Estas enfermedades se presentan en países con climas tropicales y subtropicales ${ }^{(11)}$; y también en países con climas templados ${ }^{(12)}$. Por otra parte, las nematodosis gastrointestinales en pequeños rumiantes son causantes de importantes repercusiones económicas reflejadas en una disminución del potencial productivo de los animales ${ }^{(13)}$. En un estudio realizado en México sobre el impacto económico por NGI en bovinos reportan pérdidas económicas de US\$ 445.10 millones de dólares ${ }^{(14)}$. Hasta ahora no se cuenta con un estudio sobre las pérdidas económicas que generan los NGI en pequeños rumiantes en México; sin embargo, lo citado anteriormente nos puede dar una idea del impacto negativo que causan estos parásitos en la industria ovicaprina nacional.

\section{Drogas sintéticas o antihelmínticos}

Los antihelmínticos (AH) son drogas para el control de parásitos del ganado y se clasifican de acuerdo a su modo de acción en: 1) bencimidazoles (BZ), 2) imidazotiazoles $(\mathrm{IMZ})$ y 3) lactonas macrocíclicas $(\mathrm{LM})^{(15)}$. Los BZ se unen a la subunidad alfa de la proteína $\beta$-tubulina, evitando la polimerización entre las subunidades alfa y beta, provocando que los micro túbulos no puedan formarse, ocasionando la muerte de los nematodos ${ }^{(16,17)}$. Los IMZ actúan selectivamente como agonistas colinérgicos (receptores nicotínicos) de las membranas de las células musculares de los NGI, resultando en contracción muscular y parálisis espástica ${ }^{(16)}$. Las moléculas de LM se unen selectiva e irreversiblemente a las subunidades de los canales iónicos de cloro activados por diferentes neurotransmisores (ej. glutamato), provocando la hiperpolarización de la membrana de la célula muscular o neuronal, provocando así la parálisis y expulsión de $\operatorname{los} \mathrm{NGI}^{(18)}$.

\section{Resistencia antihelmíntica}

La resistencia antihelmíntica (RA), es la disminución en la susceptibilidad de los parásitos ante una dosis de una droga que eliminaría la mayor parte de los parásitos ${ }^{(19)}$. En México, se ha notificado la presencia de RA en rebaños ovinos de Tabasco, Chiapas, Yucatán, Campeche, Tlaxcala, Puebla, y Veracruz, alcanzando también el problema a bovinos $^{(20,21,22,23)}$. Así mismo, los NGI han desarrollado mecanismos de detoxificación antihelmíntica $^{(24,25)}$. La RA en nematodos puede alterar la proteína diana y el transporte de moléculas xenobióticas como los $\mathrm{AH}$ en el nematodo por causa de proteínas transmembranales (P-glicoproteínas, P-gp), con roles de resistencia a múltiples drogas ${ }^{(16)}$. En México, se reportaron cambios de expresión relativa de genes P-gp asociados a RA entre un aislado del nematodo $H$. contortus resistente y susceptible a ivermectina (IVM), sugiriéndolos como germoplasma de referencia para el diseño de estrategias de estudio en el diagnóstico de RA y métodos de control, con la finalidad de preservar la toxicidad de los fármacos utilizados en campo para el control de los NGI. El desarrollo de la RA se debe a una interacción de diferentes factores como: la densidad de población de los NGI, el momento del tratamiento, las condiciones climáticas, entre otros; los cuales influyen en la selección de genes de resistencia ${ }^{(17,26)}$. 


\section{Consecuencias en el medio ambiente por el uso de drogas antihelmínticas}

Los AH se eliminan a través de las heces y la orina y algunos de ellos como las LM, no son biotransformados en su totalidad dentro del animal, y ponen en riesgo a microorganismos no blancos, como artrópodos benéficos ${ }^{(27)}$ o escarabajos estercoleros $^{(28)}$. Adicionalmente, los $\mathrm{AH}$ pueden contaminar los mantos freáticos $\mathrm{y}$ ocasionar un importante desequilibrio en el ecosistema acuífero. Algunas LM como la abamectina son extremadamente tóxicas para el crustáceo planctónico Daphnia magna y es altamente tóxico para otros dáfnidos y para peces ${ }^{(29)}$ y también son un riesgo en el suelo, contra organismos benéficos como artrópodos, incluyendo moscas ${ }^{(30)}$.

\section{Riesgo en salud pública por el uso de drogas antihelmínticas}

El uso excesivo de $\mathrm{AH}$ en el ganado, implica un riesgo de contaminación de la carne, leche y subproductos originando un riesgo de salud pública ${ }^{(31,32)}$. En Irlanda casi el $60 \%$ de los hatos lecheros utilizan AH de manera preventiva ${ }^{(33)}$. En Brasil, se reportó que el $17.8 \%$ de muestras de leche contenía residuos de $\operatorname{IVM}^{(34)}$. En Minas Geráis, Brasil se demostró la presencia en leche, de amino-bencimidazoles (55.42\%), levamisol (53.57\%), avermectinas (60.24\%), tiabendazol (67.47\%), moxidectina (73.49\%), triclabendazol $(45.78 \%)$ y de benzimidazoles $(6.02 \%)^{(35)}$. En México, es necesario establecer el diagnóstico de residuos de $\mathrm{AH}$ en productos diversos para constatar su inocuidad $^{(36)}$.

\section{Métodos alternativos de control de las nematodiasis del ganado}

\section{Desparasitación selectiva (FAMACHA)}

El método FAMACHA es una estrategia de desparasitación selectiva que se basa en el grado de anemia de un animal a través de la palidez de su mucosa ocular utilizando una tarjeta. Esta tarjeta consta de 5 colores que van de rojo intenso hasta pálido o blanco, donde se usa para medir en escala de 1 a 5 la coloración de la mucosa palpebral de los ovinos $^{(37)}$. Este método junto con la medición de la condición corporal y con un examen copro-parasitológico, así como del conteo fecal de huevos (CFH) permite formular un criterio de desparasitación ${ }^{(38)}$. Este método es una herramienta de gran utilidad para identificar el riesgo parasitario causado por $H$. contortus en pequeños rumiantes ${ }^{(39,40)}$; aunque, debe realizarse por un profesional capacitado, para su uso correcto. 


\section{Manejo del pastoreo}

El sistema de pastoreo rotacional (PR) se basa en dejar a los animales pastando durante 3.5 días en una área, seguido de periodos de descanso del pastizal de 31 días en condiciones tropicales, reduciendo considerablemente los NGI en ovinos y cabras ${ }^{(41)}$. En India, se ha reportado disminución en el CFH de hasta un $55.52 \%$ comparado con un esquema de pastoreo continuo $(\mathrm{PC})^{(42)}$. En otro estudio se reportó una reducción de la población de L3 de hasta en un 48.1 \% en heces; así como mejores ganancias de pesos en animales bajo un esquema $\mathrm{PR}$, comparado con animales bajo $\mathrm{PC}^{(43)}$.

\section{Estrategia nutricional basada una dieta proteica}

Se ha comprobado que dietas iso-energéticas e iso-proteicas ayudan a prevenir y controlar algunas parasitosis ${ }^{(44)}$. La proteína y energía de ese tipo de dietas contribuyen al control de NGI, al igual que la calidad y cantidad de macro y micronutrientes en la $\operatorname{dieta}^{(45)}$, fortaleciendo la inmunidad contra los nematodos ${ }^{(46)}$.

\section{Uso de plantas con actividad antihelmíntica}

Las leguminosas poseen un alto contenido de metabolitos secundarios como taninos condensados e hidrolizables, flavonoides y otros grupos de polifenoles, que representan una alternativa de control de $\mathrm{NGI}^{(47-50)}$. En México, diferentes leguminosas han dado buenos resultados contra NGI. Por ejemplo, se ha comprobado en estudios in vitro e in vivo que Leucaena leucocephala posee un importante efecto AH contra NGI del ganado $^{(51,52)}$. Otras leguminosas como las Acacias poseen derivados del ácido hidroxicinámico en sus hojas, que ejercen una potente actividad ovicida in vitro contra H. contortus, $H$. placei y Cooperia punctata ${ }^{(53,54)}$. Asimismo, en un estudio in vivo con cabras infectadas de forma artificial con $H$. contortus que recibieron el $10 \%$ de hojas deshidratadas en su dieta redujeron hasta un $70 \%$ de la eliminación de huevos de este nematodo ${ }^{(55)}$. Por otro lado, se ha demostrado que las vainas de huizache (A. farnesina) contienen flavonoides como la narigenina 7-O-(6"-galloylglucoside) que son ovicidas y larvicidas contra $H$. contortus ${ }^{(56)}$. Estas dos especies son consideradas como una fuente de forraje rico en proteína para los rumiantes ${ }^{(57,58)}$. Caesalpinia coriaria es otra leguminosa con potencial nutracéutico, cuyos frutos secos poseen actividad antimicrobiana y antihelmíntica en salud pública y en rumiantes ${ }^{(59,60,61)}$. Recientemente, se logró identificar que el ácido gálico y un derivado de tanino, aislados a partir de frutos de $C$. coriaria fueron los responsables del efecto AH contra huevos de NGI de bovinos ${ }^{(62)}$. $\mathrm{Al}$ respecto, los frutos de esta leguminosa se han incluido como parte integral en dietas para ovinos y caprinos, demostrando que no interfiere en su consumo a niveles del $2 \%$ y $10 \%$ respectivamente ${ }^{(63,64)}$. En otro estudio biodirigido utilizando la especie arbórea Prosopis laevigata se aisló e identificó al flavonoide isorhamnetin, como potente nematicida in vitro contra $H$. contortus ${ }^{(65)}$. 


\section{Vacunación}

Los antígenos (ag's) con potencial inmunoprotector obtenidos a partir de aislamientos autóctonos de los nematodos altamente patógenos, son una alternativa contra las nematodosis en rumiantes bajo condiciones de pastoreo ${ }^{(66)}$. El análisis de ag's de Haemonchus spp., es importante para el desarrollo de vacunas recombinantes contra los principales $\mathrm{NGI}^{(67)}$. En estudios recientes, se han buscado agentes inmunizantes contra NGI bajo un enfoque de control sustentable ${ }^{(68)}$, permitiendo así el desarrollo de la primera vacuna (Barbervax) contra H. contortus derivada de ag's de superficie del recubrimiento del intestino de los nematodos; sin embargo, esta vacuna solo protege parcialmente contra el nematodo $H$. contortus. Del mismo modo, se ha propuesto y evaluado la inmunización de corderos con un ag somático recombinante (rHC23) en contra de H. contortus, obteniendo una eficacia en la reducción de huevos entre un 70 y $80 \%{ }^{(69)}$. En otro estudio, se evaluó la proteína conocida como "transtiretina", derivada de productos de excreción y secreción de $H$. contortus (HcTTR) en cabras infectadas con $H$. contortus, encontrando que dos dosis de $500 \mu \mathrm{g}$ de HcTTR recombinante lograron una reducción del $\mathrm{CFH}$ del $63.7 \%$; mientras que la carga parasitaria post-mórtem se redujo en $66.4 \%$ después del desafío comparados con un grupo control ${ }^{(70)}$.

\section{Selección genética de animales resistentes}

La resistencia genética $(\mathrm{RG})$ es la variación en la respuesta inmune representada por una población de animales con la habilidad de controlar una infección o enfermedad. Es altamente dependiente de la respuesta inmune adaptativa y tiene un origen específico relacionado a un $\mathrm{gg}^{(71)}$. Se han reportado casos de resistencia ante las infecciones causadas por NGI en varias razas de ovinos; esta resistencia es mediada por la respuesta inmune adaptativa, la cual surge tras la reinfección con un determinado patógeno y está ligada al perfil genético de los animales, siendo un rasgo que puede ser transferido de los padres a su progenie ${ }^{(72)}$. Por esta razón, la RG ante los NGI es un rasgo aprovechable en la producción de pequeños rumiantes para el control de este problema, ya que a partir de la evaluación y selección de razas o cruzas de animales resistentes para programas de crianza, basados en el mejoramiento genético, se pueden potenciar los efectos de resistencia y resiliencia involucrados en el fenotipo ante este tipo de infecciones en los miembros de las generaciones futuras ${ }^{(71,73,74)}$. Para realizar una selección de animales (SA) con un fenotipo de resistencia en una población, es necesario la evaluación y medición de diversos estándares relacionados con parámetros parasitológicos, inmunológicos y de patogenicidad, dentro de las cuales están la determinación del hpg, condición corporal, porcentaje de hematocrito, la concentración de anticuerpos (IgA, IgE), el grado de eosinofilia en sangre, entre otros ${ }^{(71,73,74,75)}$. La SA con fenotipo de resistencia puede dar la pauta para mejorar la resistencia de la progenie en programas de crianza; ya que, al obtenerse una descendencia de animales resistentes, estos presentan menos nematodos adultos y por ende una menor eliminación de huevos al suelo y una menor contaminación del pasto por L $3^{(73,74)}$. Al disminuir las parasitosis en el rebaño se 
espera una mejora en la producción ganadera y una menor dependencia del uso de $\mathrm{AH}$, así como el daño a organismos benéficos por los residuos de los antiparasitarios ${ }^{(72,76,77)}$. El mejoramiento genético de pequeños rumiantes es una estrategia alternativa de control a mediano plazo de las parasitosis causadas por NGI. La selección de marcadores genéticos e identificación de posiciones genómicas en los cromosomas (locus) ligados al fenotipo son importantes para un mejor entendimiento de los mecanismos de la respuesta inmune asociada a la resistencia a $\mathrm{NGI}^{(71,76,77,78)}$.

\section{Control biológico}

Dentro de los principales enemigos naturales de los nematodos se encuentran los hongos nematófagos (HN). Estos, además de ser saprobios, son parásitos o depredadores facultativos de nematodos ${ }^{(79)}$. El HN más promisorio en el control de nematodos del ganado es Duddingtonia flagrans. Este hongo produce una gran cantidad de clamidosporas que pueden ser incorporadas en el alimento, o bien se pueden administrar oralmente en los animales en una suspensión acuosa ${ }^{(80,81,82)}$ y una vez que éstas pasan a través del tracto digestivo llegan a las heces fecales donde entran en contacto con las larvas de los nematodos, capturándolas y alimentándose de ellas, reduciendo su población dentro de un rango de entre el 70 y $90 \%{ }^{(82-85)}$. Al disminuir la población de larvas en las heces, disminuyen las infecciones y re-infecciones por el ganado ${ }^{(86)}$. Los estudios llevados a cabo por el grupo de INIFAP han demostrado una alta efectividad de esta estrategia en la reducción de la población de larvas en heces de bovinos y ovinos bajo distintas condiciones de producción, y en diferentes regiones del país; incluyendo un estudio en una unidad de producción de leche orgánica en la región de Malpaso, Chiapas $^{(80)}$. Actualmente, existen dos productos a base de formulaciones de clamidosporas del HN D. flagrans, uno en Australia, bajo el nombre de BioWorma ${ }^{(82)}$ y otro en Brasil, con el nombre de Bioverm ${ }^{(87)}$. En México, en el CENID-SAI del INIFAP actualmente se trabaja para establecer un acuerdo con una empresa para comercializar un producto a base de clamidosporas de la cepa mexicana del HN para beneficio de la actividad pecuaria.

\section{Método integrado de control}

Para lograr un control adecuado de los NGI es importante considerar el blanco de ataque de estos parásitos, basándose en el ciclo de los parásitos, tomando en cuenta que estos pueden ser encontrados básicamente en tres sitios: 1) dentro de los animales, en el tracto digestivo como larvas histiotróficas (L4), como estadios pre-adultos (L5) y como parásitos adultos, junto con los huevos que son producidos por las hembras; 2) en las heces se pueden encontrar a las fases de huevo, y fases larvarias L1 y L2 o pre-infectantes; así como las larvas infectantes (L3); 3) en el suelo y en el pasto, se encuentra a las fases L3. Con base en estos tres sitios donde se encuentran los distintos estadios de desarrollo, se propone una estrategia integral, utilizando como blancos de ataque estos sitios donde se encuentran los parásitos. En la Figura 1, se muestra un esquema representativo de los 
tres sitios "blanco" donde pueden ir dirigidas las diferentes herramientas de control para que de manera integrada se logre obtener una mayor eficacia en el control de estos parásitos.

Figura 1: Esquema representativo de la integración de los principales métodos de control de nematodos gastrointestinales de ovinos dirigidos a los distintos blancos evolutivos del parásito en sus fases endógena y exógena del ciclo biológico

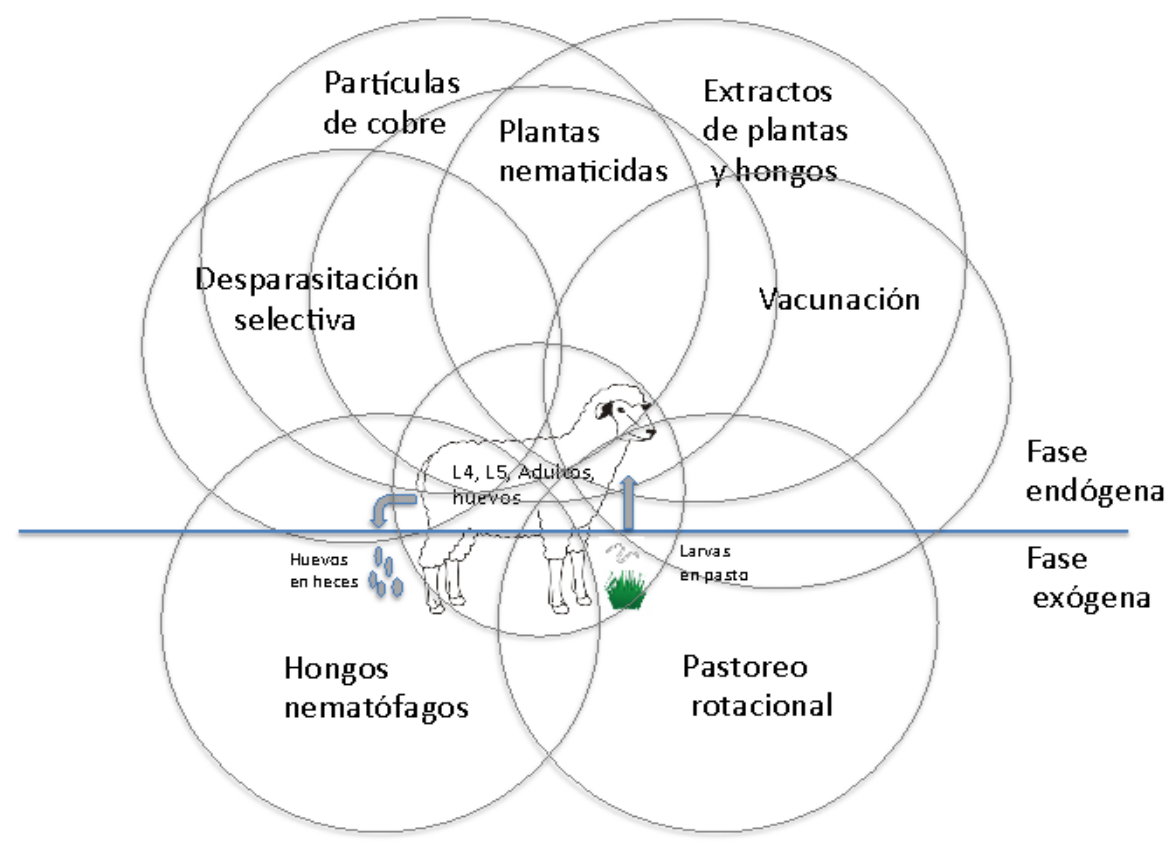

Conclusiones

Existen medidas de control probadas científicamente contra las nematodosis del rebaño que deben ser implementadas de manera integrada para mejorar su salud y su productividad, evitando el uso excesivo de AH. El método integrado de control, es una herramienta para disminuir la presentación de la RA, desde una perspectiva sustentable.

\section{Retos y Perspectivas en el control de las nematodosis del ganado en México}

La parasitología del futuro, a corto, mediano y largo plazo, enfrenta importantes retos en la búsqueda de estrategias de control diferentes al uso de AH. Es importante considerar que la gran variabilidad en la dinámica de las poblaciones de parásitos en gran medida responde a los cambios climatológicos ${ }^{(88)}$. De esta forma, la diseminación de la RA, seguida de la ineficiencia de los $\mathrm{AH}$, son una amenaza constante a los rebaños; por tal motivo se deberán diseñar estrategias para bloquear o revertir los mecanismos genómicos adaptativos de $\mathrm{RA}^{(89)}$. Nuevos ag's inmunoprotectores basados en tecnologías recombinantes deberán ser explorados para hacer más eficientes las defensas del 
$\operatorname{organismo}^{(69,70,90)}$. El uso de tecnologías sustentables deberán ser consideradas ${ }^{(91)}$, como el uso de plantas y sus metabolitos con actividad nematicida ${ }^{(92,93)}$. Por otro lado, el uso de HN para el control de parásitos en bovinos y pequeños rumiantes ${ }^{(80,81,82)}$, deberá ser implementado en México, llevándolo a nivel de comercialización e implementación en la actividad productiva para beneficio de los productores. De igual manera, el estudio de nanopartículas y de metabolitos de HN también son campos promisorios que deben ser ampliamente explorados ${ }^{(94)}$; para encontrar un control adecuado de las nematodiasis del $\operatorname{ganado}^{(95,96)}$.

\section{Contribuciones al estudio de las nematodosis del ganado}

México ha contribuido de forma significativa al quehacer científico particularmente en líneas de investigación tales como Resistencia Antihelmíntica, en la que se ha incluido el estudio del uso de herramientas moleculares para la identificación de genes marcadores de resistencia en parásitos en contra de drogas antihelmínticas ${ }^{(17,21,82)}$. Estas investigaciones se han logrado profundizar y se ha incursionado en el estudio de transcriptomas de nematodos, con la finalidad de abrir nuevas perspectivas para una posible reversión de la resistencia en los parásitos hacia los antihelmínticos; además de abordar la detección de animales resistentes a los parásitos, desde una perspectiva genética y molecular ${ }^{(78,80)}$. Otra importante línea de investigación que se ha abordado en México es el estudio de plantas y sus metabolitos con actividad nematicida contra parásitos del ganado que ha generado una importante fuente de información que dan la pauta para establecer el uso de plantas con actividad antiparasitaria en beneficio de los productores $^{(61,66,72)}$. Por otra parte, se ha generado una importante tecnología sustentable para el control de las nematodiasis en rumiantes; mediante la utilización de una cepa Mexicana (FTHO-8) de un HN de la especie Duddingtonia flagrans que es un enemigo natural de los nematodos. Las esporas de resistencia o clamidosporas de este HN han sido incorporadas en unas "galletas" o "péllets" para el ganado con lo que se consigue que al ser ingeridas por los animales, estas pasen a través del tracto digestivo y lleguen a las heces en donde germinan, colonizan las heces y forman trampas a partir de sus micelios con las que capturan, matan y se alimentan de los nematodos interrumpiendo de esta manera el ciclo biológico de estos parásitos ${ }^{(92)}$. Este es un método sustentable que ha sido demostrado de manera exitosa en distintas pruebas y bajo distintas condiciones ambientales y de manejo de los animales ${ }^{(87,88,91,93)}$. Finalmente, México ha incursionado en una línea de investigación de vanguardia sobre al estudio de las propiedades antiparasitarias de hongos comestibles y hasta ahora se han logrado importantes resultados incluyendo la identificación de metabolitos bioactivos contra estos parásitos $^{(97)}$.

\section{Agradecimientos}

Los autores expresamos nuestro agradecimiento al INIFAP y al CONACYT por los apoyos recibidos para la realización de nuestros proyectos de investigación. 


\section{Conflicto de intereses}

Los autores manifiestan que no existe ningún conflicto de intereses.

\section{Literatura citada:}

1. IICA, Carne Ovina. Caracterización del valor nutricional de alimentos. PROCITUR, IICA, Montevideo, Uruguay; 2015:158-169. http://repiica.iica.int/docs/B3885e/B3885e.pdf.

2. Tuinterfaz. Se logró reducir importaciones en $74 \%$, de 58 mil toneladas a 10 mil 379 toneladas de carne: Sagarpa. 2018. https://tuinterfaz.mx/noticias/22/10454/en-17anos-la-produccion-de-ovino-crecio-70/.

3. Agnusdei GM. Calidad nutritiva del forraje. Sitio Argentino de Producción Animal. Agromercado Temático 2007, Bs. As., 136:11-17.

4. Díaz-Sánchez CC, Jaramillo-Villanueva JL, Vargas-López S, Delgado-Alvarado A, Hernández-Mendo O, Casiano-Ventura MA. Evaluación de la rentabilidad y competitividad de los sistemas de producción de ovinos en la región de Libres, Puebla. Rev Mex Cienc Pecu 2018;9(2):273-277.

5. Rojas-Downing MM, Nejadhashemi AP, Harrigan T, Woznicki SA. Climate change and livestock: Impacts, adaptation, and mitigation. Clim Risk Managem 2017;16:145-163. doi:10.1016/j.crm.2017.02.001.

6. Craig TM. Gastrointestinal nematodes, diagnosis and control. Vet Clin North Am Food Anim Pract 2018;34(1):185-199. doi:10.1016/j.cvfa.2017.10.008.

7. Roeber F, Jex AR, Gasser RB. Impact of gastrointestinal parasitic nematodes of sheep, and the role of advanced molecular tools for exploring epidemiology and drug resistance - an Australian perspective. Parasit Vectors 2013;6(153). doi:10.1186/1756-3305-6-153.

8. Mondragón-Ancelmo J, Olmedo-Juárez A, Reyes-Guerrero DE, Ramírez-Vargas G, Ariza-Román AE, López-Arellano ME, et al. Detection of gastrointestinal nematode populations resistant to albendazole and ivermectin in sheep. Animals 2019;9:775. doi:10.3390/ani9100775.

9. López-Ruvalcava OA, González-Garduño R, Osorio-Arce MM, Aranda-Ibañez A, Díaz-Rivera P. Cargas y especies prevalentes de nematodos gastrointestinales en ovinos de pelo destinados al abasto. Rev Mex Cienc Pecu 2013;4(2):223-234.

10. Schallig HDFH. Immunological responses of sheep to Haemonchus contortus. Parasitol 2000;120(7):63-72. doi:10.1017/s003118209900579x.

11. Selemon M. Review on Control of Haemonchus contortus in sheep and goat. J Vet Med Res 2018;5(5):1139. 
12. Manninen S, Oksanen A. Haemonchosis in a sheep flock in North Finland [2010]. Acta Vet Scand 2010.https://doi.org/10.1186/1751-0147-52-S1-S19.

13. Mavrot F, Hertzberg H, Torgerson P. Effect of gastro-intestinal nematode infection on sheep performance: a systematic review and meta-analysis. Parasit Vectors 2015;8:557. https://doi.org/10.1186/s13071-015-1164-z.

14. Rodríguez-Vivas RI, Grisi L, Pérez-de León AA, Silva-Villela H, Torres-Acosta JJJ, Fragoso Sánchez H, et al. Potential economic impact assessment for cattle parasites in Mexico. Review. Rev Mex Cienc Pecu 2017;8(1):61-74.

15. Holden-Dye L, Walker RJ. Anthelmintic drugs and nematicides: studies in Caenorhabditis elegans. In: The C. elegans Research Community (ed.). WormBook; 2014. doi/10.1895/wormbook.1.143.2.

16. Kotze AC, Prichard RK. Antihelmintic resistence in Haemonchus contortus: History, mechanisms and diagnosis. In: Gasser RB, Von Samson-Himmelstjerna G. editors. Haemonchus contortus and haemonchosis - Past, present and future trends, London: Elsevier Ltd.; 2016:397-428.

17. Lanusse CE, Alvarez LI, Lifschitz AL. Gaining insights into the pharmacology of anthelmintics using Haemonchus contortus as model nematode, in: Gasser RB, Von Samson-Himmelstjerna G. editors. Haemonchus contortus and haemonchosis Past, present and future trends, London: Elsevier Ltd; 2016:465-518.

18. Laing R, Gillan V, Devaney E. Ivermectin - old drug, new tricks?, Trends Parasitol 2017;33:463-472. https://doi.org/10.1016/j.pt.2017.02.004.

19. Mphahlele M, Molefe N, Tsotetsi-Khambule A, Oriel T. Anthelmintic resistance in livestock. In: Helminthiasis, IntechOpen 2019. http://dx.doi.org/10.5772/intechopen.87124.

20. Medina P, Guevara F, La OM, Ojeda N, Reyes E. Resistencia antihelmíntica en ovinos: una revisión de informes del sureste de México y alternativas disponibles para el control de nematodos gastrointestinales. Pastos y Forrajes 2014;37(3):257263.

21. Encalada-Mena L, Tuyub-Solis H, Ramírez-Vargas G, Mendoza-de-Gives P, Aguilar-Marcelino L, López-Arellano ME. Phenotypic and genotypic characterisation of Haemonchus spp. and other gastrointestinal nematodes resistant to benzimidazole in infected calves from the tropical regions of Campeche State, Mexico, Vet Parasitol 2014;205:246-254. https://doi.org/10.1016/j.vetpar.2014.06.032.

22. González-Garduño R, Torres-Hernández G, López-Arellano ME, Mendoza-deGives P. Resistencia antihelmíntica de nematodos parásitos en ovinos, Rev Geogr Agrí 2012;48:63-74. https://www.redalyc.org/articulo.oa?id=75730739005. 
23. Alonso-Díaz MA, Arnaud-Ochoa RA, Becerra-Nava R, Torres-Acosta JF, Rodriguez-Vivas RI, Quiroz-Romero RH. Frequency of cattle farms with ivermectin resistant gastrointestinal nematodes in Veracruz, Mexico. Vet Parasitol 2015;212(3-4):439-443.

24. Lindblom TH, Dodd AK. Xenobiotic Detoxification in the nematode Caenorhabditis elegans. J Exp Zool 2006;305(9):720-730.

25. Reyes-Guerrero DE, Cedillo-Borda M, Alonso-Morales RA, Alonso-Díaz MA, Olmedo-Juárez A, Mendoza-de-Gives $\mathrm{P}$, et al. Comparative study of transcription profiles of the P-glycoprotein transporters of two Haemonchus contortus isolates: susceptible and resistant to ivermectin, Mol Biochem 2020;238(111281):1-7. https://doi.org/10.1016/j.molbiopara.2020.111281.

26. Traversa D, von Samson-Himmelstjerna G. 2016. Anthelmintic resistence in sheep gastro-intestinal strongyles in Europe. Small Rum Res 2016;135:75-80.

27. Floate KD, Wardhaugh KG, Boxall AB, Sherratt TN. Fecal residues of veterinary parasiticides: nontarget effects in the pasture environment. Annu Rev Entomol 2005;50:153-179.

28. Verdú JR, Cortez V, Martinez-Pinna J, Ortiz AJ, Lumaret JP, Lobo JM, et al. First assessment of the comparative toxicity of ivermectin and moxidectin in adult dung beetles: Sub-lethal symptoms and pre-lethal consequences. Sci Rep 2018;8(14885). doi:10.1038/s41598-018-33241-0.

29. Tišler T, Kožuh-Eržen N. Abamectin in the aquatic environment. Ecotoxicol 2006;15:495-502. https://doi.org/10.1007/s10646-006-0085-1.

30. Daeseleire E, Van Pamel E, Van Poucke C, Croubels S. Veterinary drug residues in foods. In: Schrenk D, editor. Chemical contaminants and residues in food. 1st ed. Woodhead Publishing; 2017:117-153. doi:10.1016/b978-0-08-100674-0.00006-0.

31. Beyene T. Veterinary drug residues in food-animal products: Its risk factors and potential effects on public health. J Vet Sci Tech 2015;07(01):doi:10.4172/21577579.1000285 .

32. Moreno L, Lanusse C. Veterinary drug residues in meat-related edible tissues. In: Moreno L, Lanusse C. editors. New aspects of meat quality. Elsevier: 2017:581603. doi:10.1016/b978-0-08-100593-4.00024-2.

33. Bennema SC, Vercruysse J, Morgan E, Stafford K, Hoglund J, Demeler J, et al. Epidemiology and risk factors for exposure to gastrointestinal nematodes in dairy herds in northwestern Europe. Vet Parasitol 2010;173:247-254.

34. Lobato V, Rath S. Reves FGR. Occurence of ivermectin in bovine milk from the Brazilian retail market. Food Addit Contam 2006;23:668-673. 
35. Cerqueira OPM, Souza NF, França-da-Cunha A, Almeida-Picinin LC, Leite OM, Souza RM, et al. Detection of antimicrobial and anthelmintic residues in bulk tank milk from four different mesoregions of Minas Gerais State -Brazil Minas Gerais Brasil. Arq Bras Med Vet e Zootec 2014;66(2):621-625.

36. Lourenco A, Fraga M, De Colli L, Moloney M, Danaher M, Jordan K. Determination of the presence of pathogens and anthelmintic drugs in raw milk and raw milk cheeses from small scale producers in Ireland. LWT 2020;109347. doi:10.1016/j.lwt.2020.109347.

37. Kaplan RM, Burke JM, Terril TH, Miller JE, Getz WR, Valencia E, et al. Validation of the FAMACHA ${ }^{\odot}$ eye color chart for detecting clinical anemia in sheep and goats on farms in the southern United States. Vet Parasitol 2004;123(1-2):105-120.

38. Moors E, Gauly M. Is the FAMACHA ${ }^{\odot}$ chart suitable for every breed? Correlations between FAMACHA $^{\odot}$ scores and different traits of mucosa colour in naturally parasite infected sheep breeds. Vet Parasitol 2009;166(1-2):108-111.

39. Harlow I. FAMACHA scoring to identify parasite risk in small ruminants. Farm \& Dairy 2016. https://www.farmanddairy.com/top-stories/famacha-scoring-toidentify-parasite-risk-in-small-ruminants/316777.html.

40. Gonçalves-da Silva D, Martins de Menezes B, Fernandes Bettencourt A, Frantz AC, Ribeiro-Corrêa M, Ruszkowski G, et al. Método FAMACHA® como ferramenta para verificar a infestação parasitária ocasionada por Haemonchus spp. em ovinos PubVet 2017;11(10):1015-1021. doi:10.22256/pubvet.v11n10.1015-1021.

41. Barger IA, Siale K, Banks DJD, Le Jambre LF. Rotational grazing for control of gastrointestinal nematodes of goats in a wet tropical environment. Vet Parasitol 1994;53:109-116.

42. Ram-Prasad MS, Sundaram SM, Gnanaraj PT, Bandeswaran C, Harikrishnan TJ, Sivakumar T, et al. Influence of intensive rearing and continuous and rotational grazing systems of management on parasitic load of lambs. Vet World 2019;12(8):1188-1194.

43. Devi T, Muthuramalingam T, Tensingh-Gnanaraj P, Bino-Sundar ST, SermaSaravana-Pandian A, Jemimah R. Rotational grazing pasture management system in sheep in Tamil Nadu to gain better bodyweight through the control of nematodes. J Anim Res 2019;9(3):495-497. doi:10.30954/2277-940X.03.2019.16.

44. Torres-Acosta JFJ, Sandoval-Castro CA, Hoste H, Aguilar-Caballero AJ, CámaraSarmiento R, Alonso-Díaz MA. Nutritional manipulation of sheep and goats for the control of gastrointestinal nematodes under hot humid and subhumid tropical conditions. Small Ruminant Res 2012;103:28-40. 
45. Hoste H, Torres-Acosta JFJ, Quijada J, Chan-Perez I, Dakheel MM, Kommuru DS, et al. Interactions Between nutrition and infections with Haemonchus contortus and related gastrointestinal nematodes in small ruminants. Adv Parasit 2016;93:239351. doi:10.1016/bs.apar.2016.02.025

46. Bricarello PA, Amarante AFT, Rocha RA, Cabral Filho SL, Huntley JF, Houdijk JGM, et al. Influence of dietary protein supply on resistance to experimental infections with Haemonchus contortus in Ile de France and Santa Ines lambs. Vet Parasitol 2005;134:99-109.

47. Lisonbee LD, Villalba JJ, Provenza FD, Hall JO. Tannins and self-medication: Implications for sustainable parasite control in herbivores. Behav Process 2009;82(2):184-189.

48. Williams AR, Ropiak HM, Fryganas C, Desrues O, Muller-Harvey I, Thamsborg SM. Assessment of the anthelmintic activity of medicinal plant extracts and purified condensed tannins against free-living and parasitic stages of Oesophagostomum dentatum. Parasit Vector 2014;19(7):518. doi: 10.1186/s13071-014-0518-2.

49. Zabré G, Kaboré A, Bayala B, Katiki LM, Costa-Júnior LM, Tamboura HH, et al. Comparison of the in vitro anthelmintic effects of Acacia nilotica and Acacia raddiana. Parasite 2017;24(44):1-11. https://doi.org/10.1051/parasite/2017044.

50. Brito DRB, Costa-Júnior LM, Garcia JL, Torres-Acosta JFJ, Louvandini H, CutrimJúnior JAA, et al. Supplementation with dry Mimosa caesalpiniifolia leaves can reduce the Haemonchus contortus worm burden of goats. Vet Parasitol 2018;252:47-51.

51. Mejia-Hernández P, Salem AZM, Elghandour MMMY, Cipriano-Salazar M, CruzLagunas B, Camacho LM. Anthelmintic effects of Salix babylonica L. and Leucaena leucocephala Lam. extracts in growing lambs. Trop Anim Health Product 2013;46:173-178.

52. Von Son-de Fernex E, Alonso-Díaz MÁ, Mendoza-de Gives P, Valles-de la Mora B, González-Cortazar M, Zamilpa A, et al. Elucidation of Leucaena leucocephala anthelmintic-like phytochemicals and the ultrastructural damage generated to eggs of Cooperia spp. Vet Parasitol 2015;214(1-2):89-95. doi:10.1016/j.vetpar.2015.10.005.

53. Castillo-Mitre GF, Olmedo-Juárez A, Rojo-Rubio R, Cortázar-González M, Mendoza-de Gives P, Hernández-Beteta EE, et al. Caffeoyl and coumaroyl derivatives from Acacia cochliacantha exhibit ovicidal activity against Haemonchus contortus. J Ethnopharmacol 2017;204:125-131. 
54. Olmedo-Juárez A, Rojo-Rubio R, Zamilpa A, Mendoza de Gives P, Arece-García $\mathrm{J}$, López-Arellano ME, et al. In vitro larvicidal effect of a hydroalcoholic extract from Acacia cochliacantha leaf against ruminant parasitic nematodes. Vet Res Commun 2017;41:227-232.

55. Castillo-Mitre GF, Rojo-Rubio R, Olmedo-Juárez A, Mendoza de Gives P, VázquezArmijo JF, Zamilpa A, et al. El consumo de hojas de Acacia cochliacantha reduce la eliminación de huevos de Haemonchus contortus en heces de cabritos Boer. Rev Mex Cien Pecu 2021;12(1):138-150.

56. Zarza-Albarrán MA, Olmedo-Juárez A, Rojo-Rubio R, Mendoza-de Gives P, González-Cortazar M, Tapia-Maruri D, et al. Galloyl flavonoids from Acacia farnesiana pods possess potent anthelmintic activity against Haemonchus contortus eggs and infective larvae. J Ethnopharmacol 2020;249:112402.

57. García-Winder LR, Goñi-Cedeño S, Olguin-Lara PA, Díaz-Salgado G, ArriagaJordan CM. Huizache (Acacia farnesiana) whole pods (flesh and seeds) as an alternative feed for sheep in Mexico. Trop Anim Health Prod 2009;41:1615-1621.

58. León-Castro Y, Olivares-Pérez J, Rojas-Hernández S, Villa-Mancera A, ValenciaAlmazán MT, Hernández-Castro E, et al. Effect of three fodder trees on Haemonchus contortus control and weight variations in kid. Ecosis Recur Agrop 2015;2(5):193-201.

59 Olmedo-Juárez A, Briones-Robles T, Zaragoza-Bastida A, Zamilpa A, OjedaRamírez D, Mendoza de Gives P, et al. Antibacterial activity of compounds isolated from Caesalpinia coriaria (Jacq) Willd against important bacteria in public health. Microb Pathog 2019;136:103660.

60. De Jesús-Martínez X, Olmedo-Juárez A, Olivares-Pérez J, Zamilpa A, Mendoza de Gives P, López-Arellano ME, et al. In vitro anthelmintic activity of methanolic extract from Caesalpinia coriaria J. Willd fruits against Haemonchus contortus eggs and infective larvae. Biomed Res Inter 2018;7375693. https://doi.org/10.1155/2018/7375693.

61. De Jesús-Martínez X, Olmedo-Juárez A, Rojas-Hernández S, Zamilpa A, Mendozade-Gives P, López-Arellano ME, et al. Evaluation of the hydroalcoholic extract elaborated with Caesalpinia coriaria Jacq Willd tree fruits in the control of Haemonchus contortus Rudolphi. Agrofor Syst 2020;94:1315-1321.

62. García-Hernández C, Rojo-Rubio R, Olmedo-Juárez A, Zamilpa A, Mendoza de Gives P, Antonio-Romo IA, et al. Galloyl derivatives from Caesalpinia coriaria exhibit in vitro ovicidal activity against cattle gastrointestinal parasitic nematodes. Exp Parasitol 2019;200:16-23. 
63. Sánchez N, Mendoza GD, Martínez JA, Hernández PA, Camacho-Díaz LM, LeeRangel HA, et al. Effect of Caesalpinia coriaria fruits and soybean oil on finishing lamb performance and meat characteristics. Biomed Res Int 2018;9486258. https://doi.org/10.1155/2018/9486258.

64. García-Hernández C, Olmedo-Juárez A, Mendoza de Gives P, Mondragón-Ancelmo J, Rojo-Rubio R. Efecto nutracéutico del fruto de Caesalpinia coriaria (Jacq.) Willd en cabritos infectados artificialmente con Haemonchus contortus. En: Memorias de Reunión Anual de Investigación Pecuaria 2019;1:494-496.

65. Delgado-Nuñez EJ, Zamilpa A, González-Cortazar M, Olmedo-Juárez A, CardosoTaketa A, Sánchez-Mendoza E, et al. Isorhamnetin: A nematocidal flavonoid from Prosopis laevigata leaves against Haemonchus contortus eggs and larvae. Biomolecules 2020;10:773. doi:10.3390/biom10050773.

66. Bassetto CC, Silva MRL, Newlands GFJ, Smith WD, Ratti Júnior J, Martins CL, et al. Vaccination of grazing calves with antigens from the intestinal membranes of Haemonchus contortus: effects against natural challenge with Haemonchus placei and Haemonchus similis. Int J Parasitol 2014;44:697-702. http://dx.doi.org/10.1016/j.ijpara.2014.04.010.

67. Contreras-Ochoa CO, Lagunas-Martínez A, Reyes-Guerrero DE, G.A. BautistaGarcía G, Tello-López T, González-Garduño R, et al. Excreted and secreted products $(72 / 60 \mathrm{kDa})$ from Haemonchus placei larvae induce in vitro peripheral blood mononuclear cell proliferation and actívate the expression of cytokines and FCER1A receptor. Exp Parasitol 2019;206:1-7. https://doi.org/10.1016/j.exppara.2019.107755.

68. Bassetto CC, Amarante AFT. Vaccination of sheep and cattle against haemonchosis. J Helminthol 2015;doi:10.1017/S0022149X15000279.

69. González-Sánchez ME, Cuquerella M, Alunda JM. Vaccination of lambs against Haemonchus contortus with the recombinant rHc23. Effect of adjuvant and antigen dose. PLoS ONE 2018;13(3):e0193118. https://doi.org/10.1371/ journal.pone.0193118.

70. Tian X, Lu M, Jia C, Bu Y, Aimulajiang K, Zhang Y, et al. Haemonchus contortus transthyretin domain - containing protein (HcTTR): a promising vaccine candidate against Haemonchus contortus infection. Vet Parasitol 2020;109045. doi:10.1016/j.vetpar.2020.109045

71. Maza-Lopez J, Pacheco-Armenta MJ, Reyes-Guerrero DE, Olmedo-Juárez A, Olazarán-Jenkins S, et al. Immune response related to Pelibuey sheep naturally infected with gastrointestinal nematodes in a tropical region of Mexico. Vet Parasitol Regional Stud Rep 2020;21:100422 https://doi.org/10.1016/j.vprsr.2020.100422. 
72. Preston SJM, Sandeman M, González J, Piedrafita D. Current status for gastrointestinal nematode diagnosis in small ruminants: Where are we and where are we going? J Immunol Res 2014;210350:1-12. https://doi.org/10.1155/2014/210350.

73. Estrada-Reyes Z, López-Arellano ME, Torres-Acosta F, López-Reyes A, LagunasMartínez A, Mendoza-de-Gives P, et al. Cytokine and antioxidant gene profiles from peripheral blood mononuclear cells of Pelibuey lambs after Haemonchus contortus infection. Parasite Immunol 2017;39(6):e12427. https://doi.org/10.1111/pim.12427.

74. Estrada-Reyes ZM, Tsukahara Y, Amadeu RR, Goetsch AL, Gipson TA, Sahlu T, et al. Signatures of selection for resistance to Haemonchus contortus in sheep and goats. BMC Genomics 2019;20(735):1-14. https://doi.org/10.1186/s12864-0196150-y.

75. Reyes-Guerrero DE, López-Arellano ME, González-Garduño R, Ramírez-Vargas G, Mendoza-de-Gives P, Olazarán-Jenkins S, et al. Identificación del alelo B del gen de interferón gamma asociado al rechazo de la infección por Haemonchus contortus en corderos Pelibuey. Quehacer Científico en Chiapas 2016;11(2):3-9.

76. Hill WG. Is continued genetic improvement of livestock sustainable? Genetics 2016;202:877-881. doi: 10.1534/genetics.115.186650.

77. Schultz B, Serao N, Ross JW. Genetic improvement of livestock, from conventional breeding to biotechnological approaches. In: Bazer FW, et al, editors. Animal Agriculture. USA: Academic Press 2020:393-405. https://doi.org/10.1016/B978-012-817052-6.00023-9.

78. Sallé G, Moreno C, Boitard S, Ruesche J, Tircazes-Secula A, Bouvier F, et al. Functional investigation of a QTL affecting resistance to Haemonchus contortus in sheep. Vet Res 2014;45(1):45-68.

79. Nordbring-Hertz B, Jansson HB, Tunlid A. Nematophagous fungi. eLS 2011. doi:10.1002/9780470015902.a0000374.pub3.

80. Ortíz-Pérez DO, Sánchez-Muñoz B, Nahed-Toral J, Orantes-Zebadúa MÁ, CruzLópez JL, Reyes-García ME, et al. Using Duddingtonia flagrans in calves under an organic milk farm production system in the Mexican tropics. Exp Parasitol $2017 ; 175 ; 74-82$.

81. Mendoza-de-Gives P, López-Arellano ME, Aguilar-Marcelino L, Jenkins-Olazarán $\mathrm{S}$, Reyes-Guerrero DE, Ramírez-Vargas G, et al. The nematophagous fungus Duddingtonia flagrans reduces the gastrointestinal parasitic nematode larvae population in faeces of orally treated calves maintained under tropical conditions. Dose/response assessment. Vet Parasitol 2018;15(263):66-72 doi:10.1016/j.vetpar.2018.10.001. 
82. Bampidis V, Azimonti G, Bastos ML, Christensen H, Dusemund B, Kos-Durjava $\mathrm{M}$, et al. Scientific Opinion on the safety and efficacy of BioWorma® (Duddingtonia flagrans NCIMB 30336) as a feed additive for all grazing animals. EFSA Journal 2020;18(7):6208. doi:10.2903/j.efsa.2020.6208.

83. Llerandi-Juárez RD, Mendoza-de Gives P. Resistance of chlamydospores of nematophagous fungi to the digestive processes of sheep in Mexico. J Helminthol 1998;72:155-158.

84. Mendoza-de Gives P, Flores-Crespo J, Herrera-Rodríguez D, Vázquez-Prats VM, Liébano-Hernández E, Ontiveros-Fernández GE. Biological control of Haemonchus contortus infective larvae in ovine faeces by administering an oral suspension of Duddingtonia flagrans chlamydospores to sheep. J Helminthol 1998;72:343-347.

85. Casillas-Aguilar JA, Mendoza-de-Gives P, López- Arellano ME, LiébanoHernández E. Evaluation of multinutritional pellets containing Duddingtonia flagrans chlamydospores for the control of ovine haemonchosis. Ann N Y Acad Sci 2008;1149:161-163.

86. Mendoza-de Gives P, Zapata-Nieto C, Liébano-Hernández E, López-Arellano ME, Rodríguez HD, Garduño RG. Biological control of gastrointestinal parasitic nematodes using Duddingtonia flagrans in sheep under natural conditions in Mexico. Ann N Y Acad Sci 2006;1081(1):355-359. doi:10.1196/annals.1373.050.

87. Ribeiro-Braga F, Magri-Ferraz C, da Silva NE, de Araújo VJ. Efficiency of the Bioverm (Duddingtonia flagrans) fungal formulation to conrol in vivo and in vitro of Haemonchus contortus and Strongyloides papillosus in sheep. 3 Biotech 2020;10(62). https://doi.org/10.1007/s13205-019-2042-8.

88. Sallé G, Doyle SR, Cortet J. et al. The global diversity of Haemonchus contortus is shaped by human intervention and climate. Nat Commun 2019;10(4811). https://doi.org/10.1038/s41467-019-12695-4.

89. Chaudhry U, Redman EM, Kaplan R, Yazwinski T, Sargison N, Gilleard JS. Contrasting patterns of isotype-1 $\beta$-tubulin allelic diversity in Haemonchus contortus and Haemonchus placei in the southern USA are consistent with a model of localised emergence of benzimidazole resistance. Vet Parasitol 2020;109240. doi:10.1016/j.vetpar.2020.109240 https://doi.org/10.1016/j.vetpar.2020.109240.

90. Shamim A, Sajid MK, Imran M, Saqib MN. Peptides isolation from crude somatic antigens of Haemonchus contortus through SDS- PAGE. Indian J Ani Res 2017;52(914-916).doi: https://doi.org/10.18805/ijar.v0iOF.8473.

91. Powell K, Foster C, Evans S. Environmental dangers of veterinary antiparasitic agents. Vet Rec 2018;183(19):599-600. doi:10.1136/vr.k4690. 
92. Githiori JB, Höglund J, Waller PJ. Ethnoveterinary plant preparations as livestock dewormers: practices, popular beliefs, pitfalls and prospects for the future. Anim Health Res Rev 2005;6(01):91-103. doi:10.1079/ahr2005099.

93. Minho PA, Domingues FL, Gainza AY, Figueiredo A, Boligon A, Domingues R, et al. In vitro screening of plant extract on Haemonchus contortus and Rhipicephalus (Boophilus) microplus. J Essential Oil Res 2020. doi: 10.1080/10412905.2020.1746414.

94. Magri-Ferraz C, Pinheiro CSL, Elias-de-Freitas SF, Oliveira-Souza RL, Tobias LF, Victor-de-Araújo J, et al. Effect of silver nanoparticles (AgNP's) from Duddingtonia flagranson cyathostomins larvae (subfamily: cyathostominae). J Invertebr Pathol 2020;107395. doi:10.1016/j.jip.2020.107395.

95. Degenkolb T, Vilcinskas A. Metabolites from nematophagous fungi and nematicidal natural products from fungi as an alternative for biological control. Part I: metabolites from nematophagous ascomycetes. Appl Microbiol Biot 2016;100(9):3799-3812.

96. Ocampo-Gutiérrez AY, Hernández-Velázquez VM, Aguilar-Marcelino L, CardosoTaketa A, Zamilpa A, López-Arellano ME, et al. Morphological and molecular characterization, predatory behaviour and effect of organic extracts of four nematophagous fungi from Mexico, Fungal Ecol 2021;49(101004). https://doi.org/10.1016/j.funeco.2020.101004.

97. Cruz-Arévalo J, Sánchez JE, González-Cortazar M, Zamilpa A, Andrade-Gallegos $\mathrm{HR}$, Mendoza-de-Gives P, et al. Chemical composition of an anthelmintic fraction of Pleurotus eryngii against eggs and infective larvae (L3) of Haemonchus contortus. BioMed Res Int Hindawi 2020;2020:4138950. doi: https://doi.org/10.1155/2020/4138950. 